\title{
Error propagation in the hypercycle
}

\author{
P. R. A. Campos and J. F. Fontanari \\ Instituto de Física de São Carlos, Universidade de São Paulo \\ Caixa Postal 369, 13560-970 São Carlos SP, Brazil \\ P. F. Stadler \\ Institut für Theorestische Chemie, Universität Wien \\ Währingerstraße 17, A-1090 Wien, Austria \\ The Santa Fe Institute, 1399 Hyde Park Rd., Santa Fe NM 87501, USA
}

We study analytically the steady-state regime of a network of $n$ error-prone self-replicating templates forming an asymmetric hypercycle and its error tail. We show that the existence of a master template with a higher non-catalyzed self-replicative productivity, $a$, than the error tail ensures the stability of chains in which $m<n-1$ templates coexist with the master species. The stability of these chains against the error tail is guaranteed for catalytic coupling strengths $(K)$ of order of $a$. We find that the hypercycle becomes more stable than the chains only for $K$ of order of $a^{2}$. Furthermore, we show that the minimal replication accuracy per template needed to maintain the hypercycle, the so-called error threshold, vanishes like $\sqrt{n / K}$ for large $K$ and $n \leq 4$.

PACS: $87.10 .+\mathrm{e}, 87.23 . \mathrm{Kg}$

\section{INTRODUCTION}

The limitation of the length of a genome by the replication accuracy per nucleotide $(q)$ has led to a deadlock in the theories of the origin of life based on the evolution of competing self-replicating polynucleotides. According to Eigen's quasispecies model [1,2], which may serve as a paradigm here, polynucleotides have to replicate with high accuracy in order to reach a certain length, a requirement that is impossible to fulfill without the aid of specialized catalysts. However, to build those catalysts a blueprint is necessary that amounts to a large genome (the nucleotide sequence), which itself cannot be maintained without the catalysts. In particular, for polynucleotides of fixed length $L$, the quasispecies model predicts the existence of a minimal replication accuracy per genome $Q_{c}=q_{c}^{L}$, below which the genetic information is irreversibly lost. This information crisis has been termed error threshold transition. Above $Q_{c}$ the population is composed of a master copy together with a cloud of structurally similar mutants (quasispecies) [1,2]. Equally important is the finding that, except in a trivially degenerate case, two or more quasispecies cannot coexist [3], thus precluding the coexistence of templates (i.e. polynucleotides) sufficiently different from each other to code for any useful set of catalysts. Although it has been claimed that the information crisis is not really a fundamental issue, since the error threshold transition appears only in some pathological, discontinuous replication landscapes [1], the coexistence problem seems to be more pervasive, as it is associated to the form of the growth functions in the chemical kinetics equations [5.6].

In order to circumvent the aforementioned limitations of the quasispecies model, Eigen and Schuster proposed the hypercycle [7], that is, a catalytic feedback network whereby each template helps in the replication of the next one, in a regulatory cycle closing on itself. This model has gain plausibility when the ability of polynucleotides to help propagate each other was established experimentally through the study of the catalytic activity of the RNA (ribozymes) 88.9\%. Interestingly, though the error threshold phenomenon has traditionally been considered the main motivation for the proposal of the hypercycle (see [10], for instance), most of the seminal works in this field have dealt with the coexistence issue only, as they assume perfect replication accuracy for the hypercycle elements [7, 11]. In this case an arbitrary number of templates permanently coexist in a dynamical equilibrium state; if $n>4$, however, the template concentrations vary with time [7], periodically decreasing to very small values. In practice, large hypercycles are therefore susceptible to extinction via fluctuations, see e.g. [12], hence the information gain due to the coexistence of different templates in the hypercycle may not be very impressive after all. Furthermore, we will argue in this paper that coexistence in the absence of a stable equilibrium can also be achieved by a simpler arrangement, namely, the free chains, in which the cyclic order of the catalysts is interrupted.

The effect of error-prone replication (mutation) in the hypercyclic organization was investigated by introducing a mutation field as a perturbation of the error-free kinetic equations 13]. This approach, however, is not very appropriate to study the error threshold phenomenon, since the results obtained cannot be easily compared with those of the quasispecies model. In this sense, a better approach is to assume the existence of a special class of templates with no catalytic activity, so-called error-tail, that appear as a consequence of the replication errors of the hypercycle elements 14 16. However, the particular catalytic network investigated extensively within that framework was not the hypercycle, except for a short discussion in 17], but the fully connected network in which each element helps the replication of all other elements 
of the network [15,16]. (Clearly, in the case of $n=2$ elements, these two networks become identical 14.) Such a network is more robust than the hypercycle since the malfunctioning or extinction of one of its elements does not compromise the whole network. Nevertheless, besides its aesthetic appeal, the cyclic coupling of the hypercycle seems to be more realistic [9].

The goal of this paper is to investigate analytically the steady-states of a deterministic system comprised of two parts, namely, a hypercycle made up of $n$ self-replicating templates $I_{1}, I_{2}, \ldots, I_{n}$ and its error tail $I_{e}$. These parts are coupled such that any erroneous copy of the hypercycle elements will belong to the error tail. The focus of the present analysis is on the location in the parameters space of the model (i.e. replication accuracy per template, non-catalyzed and catalyzed productivity values, and hypercycle size) of the regions of stability of the diverse possibilities of coexistence between the templates composing the hypercycle. In particular, we give emphasis to the characterization of the critical parameters at which the hypercycle becomes unstable against the error tail.

The remainder of the paper is organized as follows. In Sec. II we present the chemical kinetics equations that govern the time evolution of the system and motivate the specific choice of the parameters used throughout the paper. The fixed-points of the kinetic equations are obtained analytically in Sec. III and their stability discussed in Sec. IV. The phase-diagrams showing the regions of stability of the diverse coexistence states are presented and analyzed in Sec. V. Finally, some concluding remarks are presented in Sec. VI.

\section{THE MODEL}

We consider a system composed of a hypercycle made up of $n$ elements $I_{1}, \ldots, I_{n}$ and its error tail $I_{e}$, as illustrated in Fig. 1. In contrast to the so-called elementary hypercycle [7], we assume that the templates are capable of self-replication with productivity values $A_{i}(i=1, \ldots, n)$ and $A_{e}$. Moreover, as usual, the growth promotion of template $I_{i}$ as a result of the catalysis from template $I_{i-1}$ is measured by the kinetic constants $K_{i}$. The key ingredient in the modeling is that in both processes of growth of template $I_{i}$ the probability of success is given by the parameter $Q \in[0,1]$, so that an erroneous copy, which will then belong to the error tail, is produced with probability $1-Q$. Hence the concentrations $x_{i}(i=1, \ldots, n)$ of the hypercycle elements and the concentration $x_{e}$ of the error-tail evolve in time according to the kinetic equations

$$
\dot{x}_{i}=x_{i}\left(A_{i} Q+K_{i} x_{i-1} Q-\Phi\right) \quad i=1, \ldots, n
$$

and

$$
\dot{x}_{e}=x_{e}\left(A_{e}-\Phi\right)+(1-Q) \sum_{i=1}^{n} x_{i}\left(A_{i}+K_{i} x_{i-1}\right)
$$

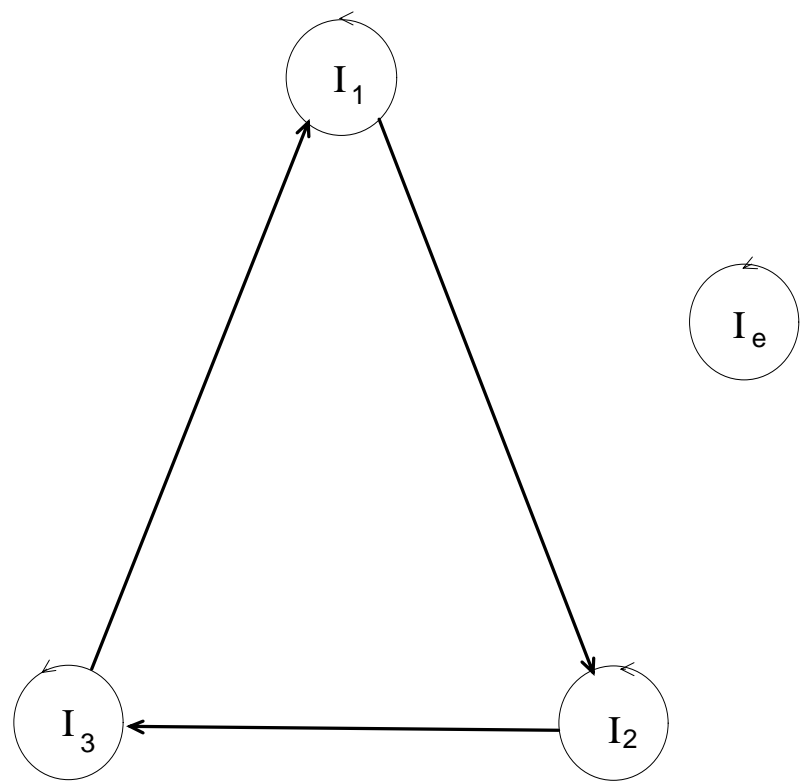

FIG. 1. The system composed of a hypercycle of size $n=3$ and its error tail $I_{e}$. The thin arrows represent the non-catalyzed self-replication reactions and the thick arrows represent the self-replication catalytically assisted by the neighbor template

where $x_{0} \equiv x_{n}$ and

$$
\Phi=\sum_{i=1}^{n} x_{i}\left(A_{i}+K_{i} x_{i-1}\right)+A_{e} x_{e}
$$

is a dilution flux that keeps the total concentration constant, i.e., $\sum_{i=1}^{n} \dot{x}_{i}+\dot{x}_{e}=0$. As usual, the dot denotes a time derivative. Henceforth we will assume that [15.16]

$$
\sum_{i=1}^{n} x_{i}+x_{e}=1
$$

Clearly, this formulation is equivalent to considering polynucleotides of length $L \rightarrow \infty$ whose replication accuracy per nucleotide $q$ goes to 1 such that the replication accuracy per genome is finite, i.e. $q^{L} \rightarrow Q$. In this limit, the back-mutations from the error-tail elements to the templates that compose the hypercycle, as well as the mutations between those templates, can be safely neglected. Hence, mutations can only increase the concentration of the elements in the error-tail. The advantage of working in this limit is that the error threshold transition can be precisely located by determining the value of $Q$ at which the concentration of a relevant template vanishes. For finite $L$, as well as for finite population sizes, the characterization of this transition is more involved, being achieved through the use of finite-size scaling techniques [18].

In this work we consider the single-sharp-peak replication landscape [1,2], in which we ascribe the productivity value $A_{1}=a>1$ to the so-called master template 
$I_{1}$ and $A_{i}=A_{e}=1$ to the $n-1$ other elements of the hypercycle as well as to the error-tail. Also, for the sake of simplicity we set $K_{i}=K$ for all $i$. The motivation for this particular choice of parameters is the observation that the emergence of the hypercycle requires both spatial and temporal coexistence of the templates forming the network, and this can be achieved by a quasispecies distribution, which guarantees the coexistence of the master template and its close mutants, despite the purely competitive character of the quasispecies model [19]. Once the coexistence is established, the appearance of catalytic couplings between the templates is not a very unlike event. Of course, as soon as those cooperative couplings become sufficiently strong to balance the competition imposed by the constant concentration constraint, the mutants will certainly depart from the master template due to the relentless pressure of the mutations, so that no trace will remain of the original quasispecies distribution.

\section{FIXED POINTS}

Let us distinguish between surviving templates $x_{i}>$ 0 and extinct templates $x_{i}=0$. A survivor $I_{j}$ is said isolated if $x_{j-1}=x_{j+1}=0$. Hence,

$$
\dot{x}_{j}=x_{j}(Q-\Phi) \quad j>1
$$

and

$$
\dot{x}_{e}=x_{e}(1-\Phi)+(1-Q)\left[x_{1}(a-1)+1+K \sum_{i \neq j, j+1} x_{i} x_{i-1}\right] .
$$

In the steady-state regime, Eq. (5) yields $\Phi=Q$ which, for $Q<1$, is incompatible with Eq. (6), since the term within brackets in this equation is positive. Therefore, all isolated survivors with the exception of the master template are unstable against the error tail. Next consider the following chain of surviving templates:

$$
\begin{gathered}
\dot{x}_{i}=x_{i}(Q-\Phi) \\
\dot{x}_{i+1}=x_{i+1}\left(Q+K Q x_{i}-\Phi\right) \\
\dot{x}_{i+2}=x_{i+2}\left(Q+K Q x_{i+1}-\Phi\right) \\
\vdots \\
\dot{x}_{k}=x_{k}\left(Q+K Q x_{k-1}-\Phi\right)
\end{gathered}
$$

which does not contain $x_{1}$. Again, in the steady-state regime the first equation yields $\Phi=Q$, implying $K Q x_{i}=$
0, i.e., $x_{i}=0$. So, there is no fixed point corresponding to such a chain. Any chain of survivors therefore must start with template $n$ or 1 . In the first case we get $\Phi=Q$ from $\dot{x}_{n}=0$ and $a Q+K Q x_{n}=Q$ from $\dot{x}_{1}=0$, yielding $x_{n}=(1-a) / K<0$, which rules out this possibility. The equilibria of interest for our study thus are either the interior equilibrium in which all templates survive, or a fixed point that corresponds to a chain of survivors beginning with $I_{1}$.

Accordingly, we define a $m$-coexistence state as the $n$ component template vector $\mathbf{x}=\left(x_{1}, x_{2}, \ldots, x_{n}\right)$ in which the first $m$ components are strictly positive and the rest are equal to zero. Clearly, given the template vector $\mathbf{x}$ the concentration of the error tail $x_{e}$ is determined by the constraint (画. In the following we solve analytically the kinetic equations in the steady-state regime $\dot{x}_{i}=0 \forall i$.

The simplest fixed point is the zero-coexistence state $(m=0)$ which corresponds to the solution $x_{1}=\ldots=$ $x_{n}=0$ and $x_{e}=1$, existing for the complete range of parameter values.

In the case of chains, i.e. $0<m<n$, the steady-state solutions of Eqns. (11) and (2) are straightforward. In fact, since $x_{n}=0$ by definition, we get $\Phi=a Q$ from $\dot{x}_{1}=0$ which then yields

$$
x_{1}=x_{2}=\ldots=x_{m-1}=\frac{a-1}{K} .
$$

Next we insert this result in Eq. (3) to obtain

$$
x_{m}=\frac{Q a-1}{a-1}-(m-1) \frac{a-1}{K} .
$$

However, since $x_{i} \in(0,1) \forall i$, this solution is physically meaningful in the region $K>a-1$ and $Q>Q_{m}$ where

$$
Q_{m}=\frac{1}{a}+\frac{1}{K a}(m-1)(a-1)^{2} .
$$

We note that the 1-coexistence state (quasispecies) is obtained by setting $m=1$ in Eq. (13) and its region of existence is simply $Q>1 / a$, since the other condition, namely $K>a-1$, is derived by considering the other templates in the chain. In fact, this very simple result quantifies nicely the notion that the cooperative couplings must reach a certain minimum strength so as to balance the competition between templates.

The analysis of the hypercycle, i.e. $m=n$, is a little more involved. ¿From $\dot{x}_{2}=0$ we get $\Phi-Q=K Q x_{1}$ which, inserted in the equations $\dot{x}_{3}=\ldots=\dot{x}_{n}=0$, yields $x_{1}=x_{2}=\ldots=x_{n-1}$ and

$$
x_{n}=x_{1}-\frac{a-1}{K} .
$$

Finally, using these results in Eq. (3) we find that $x_{1}$ is given by the roots of the quadratic equation

$$
n K x_{1}^{2}-(K Q+a-1) x_{1}+1-Q=0 .
$$


For $K<(a-1)^{2} / 4 n$, this equation has real roots for all $Q \geq 0$, otherwise it has real roots for $Q \geq Q_{h}$ where $Q_{h}$ is the unique positive root of the equation

$$
K^{2} Q_{h}^{2}+2 K(a-1+2 n) Q_{h}+(a-1)^{2}-4 n K=0 .
$$

In particular, for large $K$ we find $Q_{h} \approx 2 \sqrt{n / K}$. Furthermore, it can be easily seen from Eqns. (15) and (16) that $x_{n}$ vanishes at $Q=Q_{n}$ with $Q_{n}$ given in Eq. (14). To understand the role of $Q_{h}$ and $Q_{n}$ (we note that $Q_{n} \geq Q_{h}$ ) in delimiting the region of existence of the $n$-coexistence state we must look at the behavior of the two real roots of Eq. (16). Let us denote them by $x_{1}^{+}$and $x_{1}^{-}$with $x_{1}^{+} \geq x_{1}^{-}$, which, according to Eq. (15), correspond to $x_{n}^{+}$and $x_{n}^{-}$, respectively. Of course, these roots become identical at $Q=Q_{h}$ and so the two solutions for $x_{n}$ will vanish simultaneously only at the value of $K=K_{h}$ at which $Q_{h}$ equals $Q_{n}$. Explicitly, we obtain

$$
K_{h}=(a-1)[n(a+1)-1] .
$$

by inserting Eq. (14) into Eq. (17). Although both roots $x_{1}^{+}$and $x_{1}^{-}$are in the simplex $(0,1)$, this is not so for $x_{n}^{+}$and $x_{n}^{-}$. In particular, for $K<K_{h}$ both concentrations are negative within the range $Q_{h} \leq Q<Q_{n}$. However, while $x_{n}^{+}$becomes positive for $Q>Q_{n}$ (it vanishes at $\left.Q_{n}\right), x_{n}^{-}$remains always negative. Since $K_{h}>(a-1)^{2} / 4 n$ the same conclusion holds in the range $K<(a-1)^{2} / 4 n$ as well, provided we define $Q_{h}=0$ in this region. The situation is reversed for $K>K_{h}$ : both concentrations are positive within the range $Q_{h} \leq Q<Q_{n}$, but now it is $x_{n}^{-}$that vanishes at $Q_{n}$ and becomes negative for $Q>Q_{n}$ while $x_{n}^{+}$remains always positive. Despite the small region in the parameters space where the root $x_{1}^{-}$yields concentrations inside the simplex, the linear stability analysis discussed in the sequel indicates that this solution is always unstable, so we only need to consider the root $x_{1}^{+}$. Thus the range of existence of the hypercycle fixed point $m=n$ is $Q \geq Q_{n}$ if $K \leq K_{h}$ and $Q \geq Q_{h}$ if $K>K_{h}$.

In models without error-tail, i.e., pure replicator equations a much stronger statement on coexistence is possible. The "time average theorem" 20] states that if there is a trajectory along which a certain subset of templates $J$ survives, then there is a fixed point with exactly the $J$-coordinates non-zero. While we have not been able to prove the "time average theorem" in full generality for Eqns. (11) and (2), it is easily verified for free chains. Hence, if there is no $m$-coexistence equilibrium, then there is no trajectory at all along which the templates $I_{1}$ through $I_{m}$ survive.

Hitherto we have determined the ranges of the parameter $Q$ where the $m$-coexistence states are physically meaningful, in the sense that $x_{i} \in(0,1) \forall i$. The next step is to find the regions where these states are locally stable.

\section{STABILITY ANALYSIS}

In order to perform a standard linear stability analysis of the fixed points obtained in the previous section, it is convenient to rewrite the kinetic equations (11) and (2) as follows

$$
\dot{x}_{i}=x_{i} F_{i}(\mathbf{x}) \quad i=1, \ldots, n
$$

where

$$
F_{i}(\mathbf{x})=A_{i} Q+K Q x_{i-1}-A_{e}-\sum_{j} x_{j}\left(A_{j}-A_{e}+K x_{j-1}\right)
$$

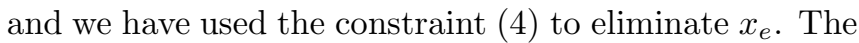
stability of a fixed point is ensured provided that the real parts of all eigenvalues of the $n \times n$ Jacobian $\mathcal{J}$ are negative. In our case the elements of the Jacobian are given by

$$
J_{i j}=\delta_{i j} F_{i}+x_{i} \frac{\partial F_{i}}{\partial x_{j}} \quad i, j=1, \ldots, n .
$$

The evaluation of the eigenvalues is simple only for the zero-coexistence state, since in this case the Jacobian is diagonal with elements $J_{11}=a Q-1$ and $J_{i i}=Q-1, i>$ 1. Therefore this steady state becomes unstable for $Q>1 / a$, which coincides with the lowest replication accuracy required for the existence of the 1-coexistence state. However, for a general $m$-coexistence state we have to resort to a numerical evaluation of the Jacobian eigenvalues.

Fortunately, in the case of chains $0<m<n$ there is an alternative way to look at the stability of the fixed points, as hinted by the stability analysis of the zero-coexistence state, which becomes unstable due to the emergence of the 1-coexistence state. In fact, it can be easily seen that any perturbation of the $m$-coexistence fixed point which makes the concentration $x_{m+1}$ non-zero will be amplified if $A_{m+1} Q+K Q x_{m}-\Phi$ is positive. For $m>0$ we use $\Phi=a Q$ and $A_{m+1}=1$ together with the value of $x_{m}$ given in Eq. (13) to obtain the following (necessary) condition for the stability of the $m$-coexistence state,

$$
Q<Q_{m+1} \quad m>0,
$$

with $Q_{m}$ given in Eq. (14). Hence the maximum value of $Q$ allowed for the stability of the $m$-coexistence state coincides with the minimum $Q$ required for the existence of the $(m+1)$-coexistence state. Interestingly, though for $m=0$ we have $\Phi=1, A_{1}=a$ and $x_{0}=0$, condition (22) holds true in this case too.

At this point two caveats are in order. First, the entire argument leading to the stability condition (22) is flawed if the $(m+1)$-coexistence state happens to be unstable. Therefore, we must guarantee via the numerical evaluation of the Jacobian eigenvalues that the $l$-coexistence 
state is stable before using that condition to study the stability of chains with $m<l$. In particular, we have carried out the numerical analysis for the hypercycle solution $l=n$ and found the following general results:

(i) For $n \leq 4$, it is always stable;

(ii) for $n=5$, it is stable in a very small range of $Q$ above $Q_{5}$; and

(iii) for $n \geq 6$, it is always unstable.

Second, the derivation of the stability condition (22) is based on the analysis of a single eigenvalue of the Jacobian and so it does not yield a sufficient condition for the stability of the fixed points. Nevertheless, we have verified through the numerical evaluation of all $n$ eigenvalues that, provided the $(m+1)$-coexistence state is stable, the eigenvalue associated to fluctuations leading to an increase of the chain length is the first one to become positive.

\section{DISCUSSION}

Combining the existence and the stability results derived in the previous sections we can draw the phasediagrams in the plane $(K, Q)$ for fixed $a$ and $n$. In particular, for $n \leq 4$ the $m$-coexistence state is stable within the interval

$$
Q_{m}<Q<Q_{m+1} \quad m<n
$$

with $Q_{m}$ given in Eq. (14), provided that $K>a-1$. Interestingly, for fixed $Q$, Eq. (23) shows that the increment $\delta K$ in the catalytic coupling needed to incorporate a new template into the chain is

$$
\delta K=\frac{(a-1)^{2}}{a Q-1},
$$

regardless the number of elements in the chain. The case $K<a-1$, for which no chains with $m>1$ are allowed, does not require any special consideration. In fact, we find that the only stable states are the zero-coexistence $(Q<1 / a)$ and the 1 -coexistence $(1 / a \leq Q \leq 1)$ states. However, since $Q_{2} \leq 1$ only for $K \geq a-1$, this result is consistent with Eq. (23).

The $n$-coexistence state (i.e., the hypercycle solution) is stable for $Q>Q_{n}$ if $K \leq K_{h}$ and for $Q>Q_{h}$, otherwise, where $K_{h}$ and $Q_{h}$ are given by Eqns. (18) and (17), respectively. We define the error threshold of the hypercycle as the value of the replication accuracy $Q$ that delimits the region of stability of the $n$-coexistence state. The phase diagram for $a=10$ and $n=4$ shown in Fig. 2 illustrates the major role played by $K_{h}$ in the hypercyclic organization: only for $K>K_{h}$ the hypercycle becomes more stable than a chain of the same length. Another important quantity is the value of $K$, denoted by $K_{c}$, at

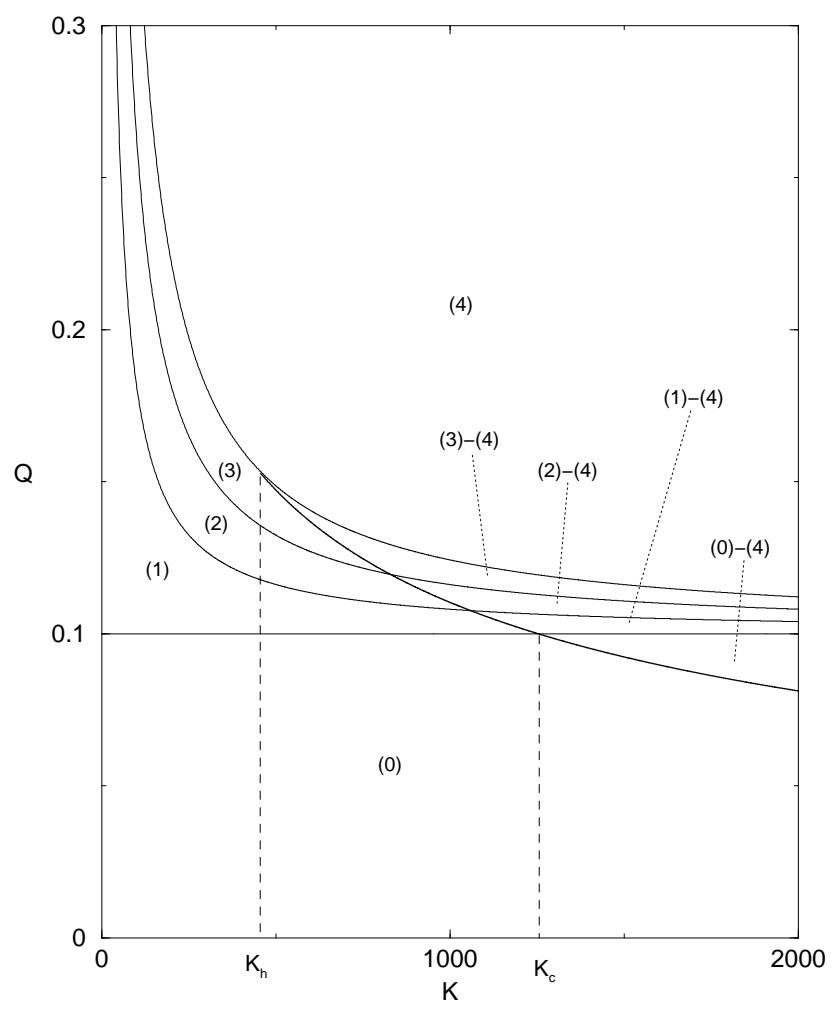

FIG. 2. Phase-diagram in the space $(K, Q)$ for $n=4$ and $a=10$ showing the regions of stability of the diverse coexistence states. The numbers between parentheses indicate the number of coexisting templates. Regions of bistability appear for $K>K_{h}$. The thin lines are (from bottom to top) $Q_{1}, Q_{2}, Q_{3}$ and $Q_{4}$. The thick line is $Q_{h}$.

which $Q_{h}$ equals $1 / a$, the minimal replication accuracy of the quasispecies. It is given by

$$
K_{c}=a(a-1)[2 n-1+\sqrt{n(n-1)}] .
$$

Beyond this value the error threshold of the hypercycle $Q_{h}$ is smaller than that of the quasispecies. Moreover, as mentioned before, for large $K$, it vanishes like $1 / \sqrt{K}$. A rather frustrating aspect of $K_{h}$ and $K_{c}$ is that both are of order $a^{2}$, indicating then that the productivity of catalytically assisted self-replication is much larger than that of non-catalyzed self-replication. While this is obviously true for biochemical catalysis, it is difficult to argue for the existence of such efficient catalysts in prebiotic conditions. On the other hand, we can take a different, more optimistic viewpoint and argue that modern biochemical catalysts (enzymes) are so efficient because their precursors had to satisfy the stringent conditions imposed by surpassing $K_{h}$.

In Fig. 3 we present the phase-diagram for $n=5$. The main difference from the previous figure is that the 5coexistence state is stable only within the thin region between $Q_{5}$ and the dashed curve, obtained through the numerical evaluation of the Jacobian eigenvalues. As these curves intersect at some $K \leq K_{h}$, the 5 -membered hypercycle is not very interesting, since it has the same 


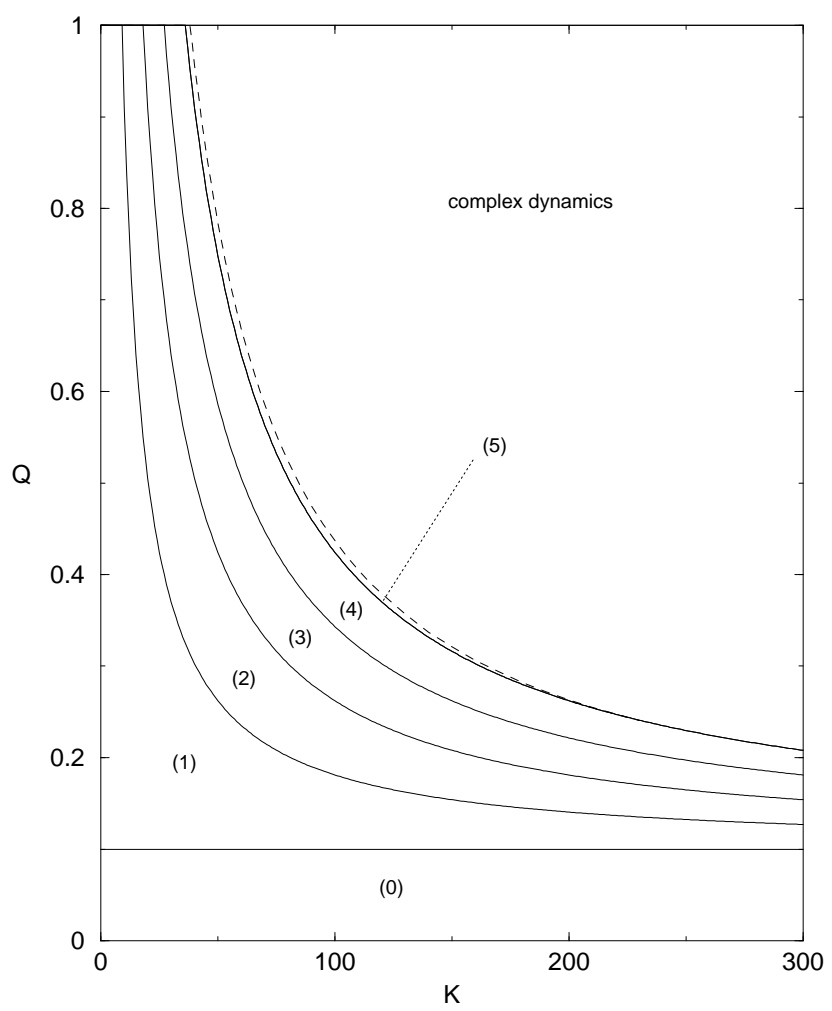

FIG. 3. Same as Fig. 1 but for $n=5$. There are no stable fixed points above the dashed curve. The solid curves are (from bottom to top) $Q_{1}, Q_{2}, Q_{3}, Q_{4}$, and $Q_{5}$.

characteristics of a chain of length $m=5$. To confirm this result we have carried out the numerical integration of the kinetic equations using the ninetieth-order Runge-Kutta method. The results are shown in Fig. 4, which illustrates the time evolution of the concentrations $x_{i}(i=1, \ldots, 5)$ inside and outside the region of stability. Although the behavior pattern in the region of instability seems periodic, we have not explored completely the space of parameters to discard the existence of chaotic behavior. Hence we use the term complex dynamics to label this region in Fig. 3. We note that the phasediagram shown in this figure describes also the regions of stability of hypercycles and chains of size $n \geq 5$, since $m$-coexistence states with $m>5$ are always unstable.

An interesting limiting case which deserves special attention is the symmetric hypercycle $(a=1)$. According to the argument put forward in the beginning of Sec. III, the only fixed points in this case are the zero-coexistence state and the hypercycle, i.e., chains are not allowed. Moreover, Eq. (15) yields $x_{1}=x_{2}=\ldots=x_{n}$ where $x_{1}$ is given by Eq. (16) with $a$ replaced by 1 . The analysis of the roots of that quadratic equation and the numerical evaluation of the Jacobian eigenvalues yield that the symmetric hypercycle is stable for

$$
K>\frac{4 n}{Q^{2}}(1-Q),
$$

provided that $n \leq 4$. The region of stability observed
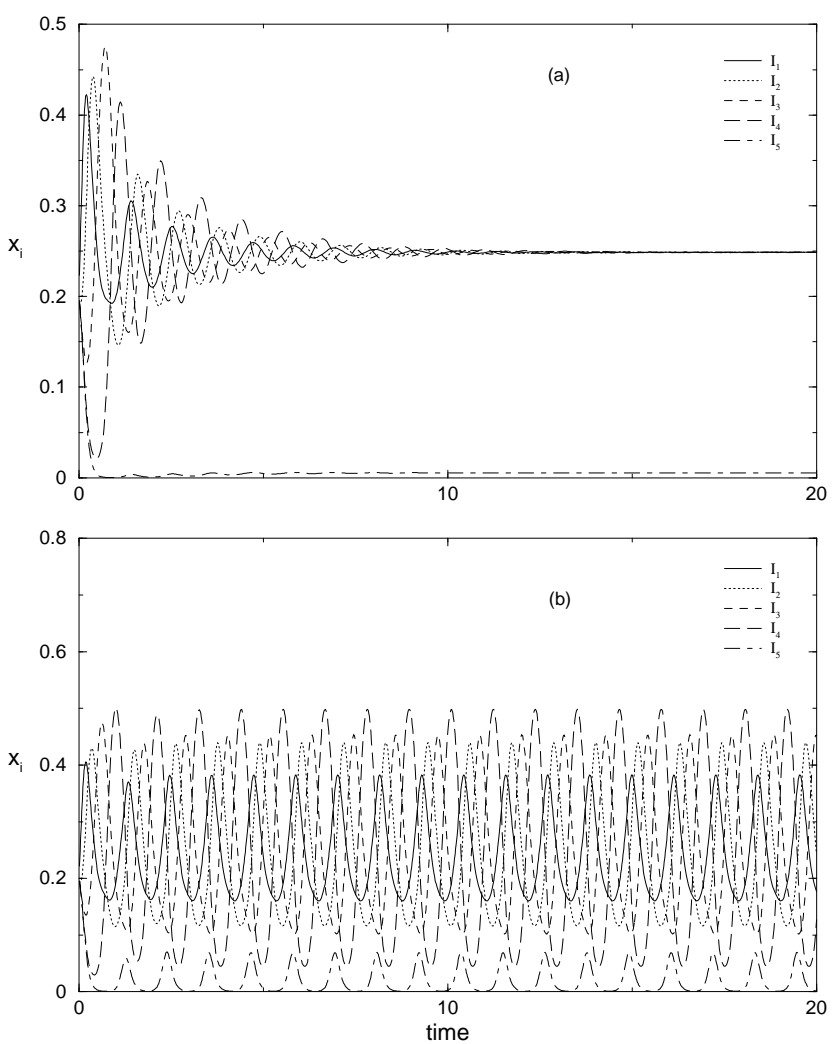

FIG. 4. Time evolution of the five concentrations of the templates composing a hypercycle of size $n=5$ for $a=10$, $Q=1$, and (a) $K=37$ (inside the region of stability) and (b) $K=40$ (outside the region of stability). The initial state is $x_{i}(0)=0.2 \forall i$.

in Fig. 3 for the 5-coexistence state does not appear in the symmetric case $a=1$, so it must be a consequence of the asymmetry in the productivity values of the non-catalyzed self-replication reaction. We note that, differently from the asymmetric case $(a>1)$, the zerocoexistence state is always stable.

For the sake of completeness, we present some results on the elementary hypercycle $\left(A_{i}=0, i=1, \ldots, n\right)$ coupled to an error tail $\left(A_{e}=1\right)$ via the imperfect catalytically assisted self-replication. Inserting these parameters into Eq. (20) and setting $F_{i}=0 \forall i$ yields $x_{1}=\ldots=x_{n}$ with $x_{1}$ given by the larger root of the quadratic equation

$$
K n x_{1}^{2}-(n+K Q) x_{1}+1=0,
$$

since we have verified that the smaller root is always unstable. As in the symmetric case discussed above, for $n \leq 4$ the stability condition coincides with the condition for real $x_{1}$, namely,

$$
Q \geq 2 \sqrt{\frac{n}{K}}-\frac{n}{K} .
$$

Thus the term in the right hand side of this inequality yields the error threshold of the elementary hypercycle. 


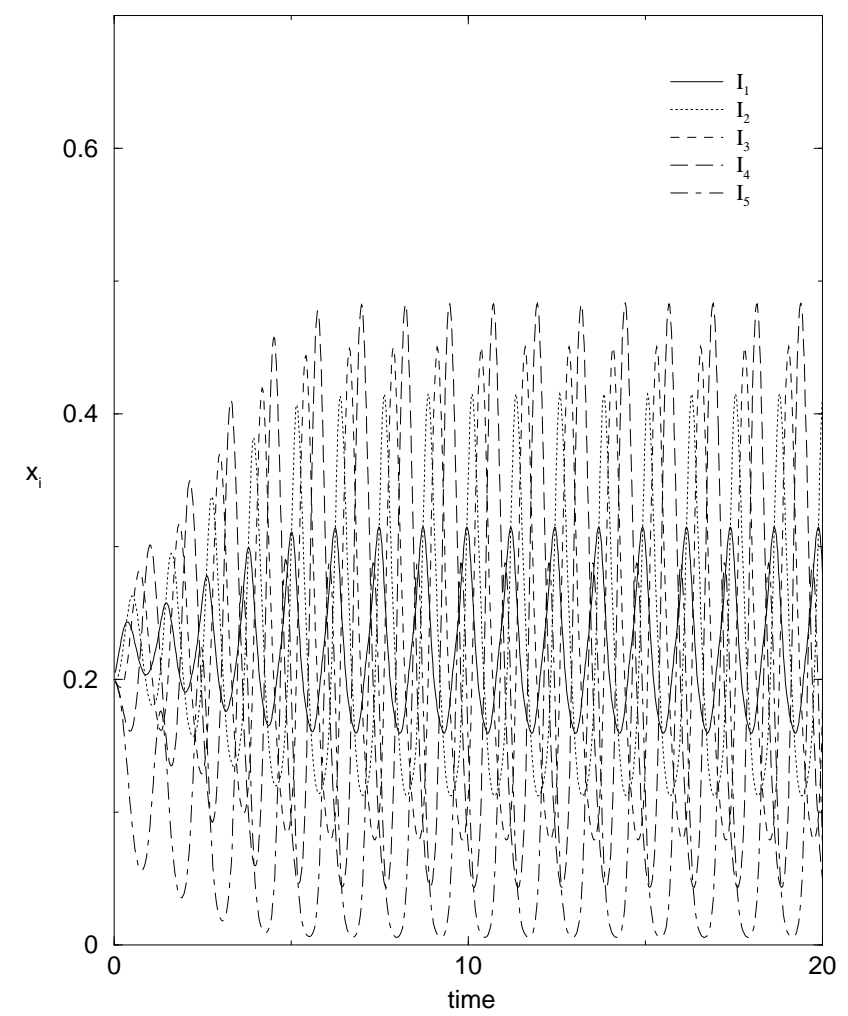

FIG. 5. Time evolution of the five concentrations of the templates composing a free chain of size $n=5$. The parameters and initial state are the same as for Fig. 1(b).

Before concluding this section, we must note that the chains of size $m$ considered hitherto are bonded in a hypercycle of size $n>m$. We could study free chains of size $n$ as well by simply setting $x_{0}=0$ in the kinetic equations (1) and (2). Not surprisingly, the results are essentially the same as for bonded chains, with $Q_{m}$ playing a similar fundamental role in delimiting the regions of stability of the shorter chains $(m<n)$. Although a full discussion of the stability of the complete chain $(m=n)$ is beyond the scope of this paper, we point out that free chains with $n>4$ are always unstable. Moreover, as illustrated in Fig. 周, the oscillatory behavior pattern of the template concentrations, which ensues a dynamic coexistence among all templates in the chain, is similar to that

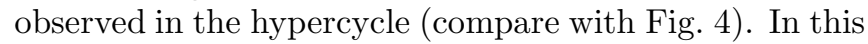
sense, the free chains seem as good as the hypercycle to attain that kind of coexistence. However, we must emphasize that, for sufficiently large $K$ (i.e. $K>K_{h}$ ), the fixed points describing the coexistence of $n \leq 4$ templates in a hypercycle are much more robust against replication errors than their counterparts in the free chains.

\section{CONCLUSION}

Our study of the steady states of an asymmetric hypercycle composed of $n$ error-prone self-replicating templates indicates that, for $n \leq 4$, the error threshold of the hypercycle $\left(Q_{h}\right)$ becomes smaller than that of the quasispecies $\left(Q_{1}=1 / a\right)$ for catalytic couplings $(K)$ of order of $a^{2}$, where $a$ is the productivity value of the master template. In particular, $Q_{h}$ vanishes like $\sqrt{n / K}$ for large $K$. Perhaps, even more important is our finding that the asymmetry in the non-catalyzed self-replication reaction $(a>1)$ entails the existence of chains of size $n \leq 5$. We note that these chains are unstable in the symmetric hypercycle as well as in the fully connected network [15. 16 .

Adding to the scenario for the emergence of the hypercycle described in Sec. II, which starts with an isolated quasispecies, our results indicate that the chains may well be the next step in this complex evolutionary process. In fact, according to Eq. (24) the strengths of the catalytic couplings needed to form a chain are of order $a$, while the hypercycle only acquires its desirable stability characteristics for strengths of order $a^{2}$ (see Eq. (18)). Although we realize that an evolutionary step leading from chains to hypercycles is still a major one, it is certainly much more plausible than a direct transition from quasispecies to hypercycle. In any event, we think that the emergence of the hypercycle can be explained as a series of plausible smooth transitions, without need to postulating the hypercycle as an unique event in prebiotic evolution. In this vein, this work represents a modest first step to tackle this fundamental problem within a firm basis.

To conclude we must mention an alternative resolution for the information crisis in prebiotic evolution which has received some attention recently [21], namely, the stochastic corrector model [22]. This model builds on ideas of the classical group selection theory for the evolution of altruism [23], since it considers replicative templates competing inside replicative compartments, whose selective values depend on their template composition. However, the chemical kinetics equations governing the dynamics of the templates inside the compartments display a non-physical (non-integer exponents) dependence of growth on template concentrations. It seems to us that this basic assumption of the stochastic corrector model must be relaxed, or at least justified, before it can be considered an important alternative to the more traditional approach based on the hypercycle and its variants.

P.R.A.C. thanks Prof. P. Schuster and Prof. P. F. Stadler for their kind hospitality at the Institut für Theorestische Chemie, where part of his work was done, and Prof. P. E. Phillipson for illuminating discussions. The work of J. F. F. was supported in part by Conselho Nacional de Desenvolvimento Científico e Tecnológico (CNPq). The work of P.F.S. was supported in part by the Austrian Fonds zur Förderung der Wissenschaftlichen Forschung, Proj. No. 13093-GEN. P.R.A.C. is supported by FAPESP. 
[1] M. Eigen, Naturwissenschaften 58, 465 (1971).

[2] M. Eigen, J. McCaskill and P. Schuster, Adv. Chem. Phys. 75, 149 (1989).

[3] J. Swetina and P. Schuster, Biophys. Chem. 16, 329 (1982).

[4] T. Wiehe, Genet. Res. 69, 127 (1997).

[5] E. Szathmáry, TREE 6, 366 (1991).

[6] P. R. Wills, S. A. Kauffman, B. M. R. Stadler and P. F. Stadler, Bull. Math. Biol. 60, 1073 (1998)

[7] M. Eigen and P. Schuster, Naturwissenchaften 65, 7 (1978).

[8] J. A. Doudna and J. W. Szostak, Nature 339, 519 (1989).

[9] M. Eigen, C. K. Biebricher, M. Gebinoga and W. C. Gardiner, Biochemistry 30, 1105 (1991).

[10] J. Maynard Smith, Nature 280, 445 (1979).

[11] J. Hofbauer, P. Schuster, K. Sigmund and R. Wolff, SIAM J. Appl. Math. 38, 282 (1980).

[12] J. C. Nuño and P. Tarazona, Bull. Math. Biol. 56, 875 (1994).

[13] P. F. Stadler and P. Schuster, J. Math. Biol. 30, 597 (1992).

[14] A. García-Tejedor, F. Morán and F. Montero, J. Theor. Biol. 127, 393 (1987).

[15] J. C. Nuño, M. A. Andrade, F. Morán and F. Montero, Bull. Math. Biol. 55, 385 (1993).

[16] J. C. Nuño, M. A. Andrade and F. Montero, Bull. Math. Biol. 55, 417 (1993).

[17] P. F. Stadler and J. C. Nuño, Math. Biosci. 122, 127 (1994)

[18] P. R. A. Campos and J. F. Fontanari, Phys. Rev. E 58, 2664 (1998); J. Phys. A 32, L1 (1999).

[19] A. García-Tejedor, A. R. Castaño, F. Morán and F. Montero, J. Mol. Evol. 26, 294 (1987).

[20] J. Hofbauer and K. Sigmund, Dynamical Systems and the Theory of Evolution (Cambridge Univ. Press, Cambridge UK, 1988)

[21] J. Maynard Smith and E. Szathmáry, The Major Transitions in Evolution (Freeman, Oxford, 1995).

[22] E. Szathmáry and L. Demeter, J. Theor. Biol. 128, 463 (1987).

[23] S. A. Boorman and P. R. Levitt, The Genetics of Altruism (Academic Press, New York, 1980). 